\title{
Effects of evodiamine on PI3K/Akt and MAPK/ERK signaling pathways in pancreatic cancer cells
}

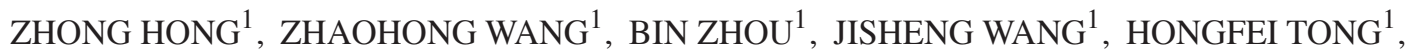 \\ YI LIAO $^{1}$, PENG ZHENG ${ }^{1}$, MUHAMMAD BABAR JAMSHED ${ }^{2}$, QIYU ZHANG ${ }^{1,2}$ and HUI CHEN ${ }^{1}$ \\ ${ }^{1}$ Department of Hepatobiliary and Pancreatic Surgery, The Second Affiliated Hospital and Yuying Children's Hospital, \\ Wenzhou Medical University, Wenzhou, Zhejiang 325035; ${ }^{2}$ Department of Hepatobiliary Surgery, \\ The First Affiliated Hospital of Wenzhou Medical University, Wenzhou, Zhejiang 325000, P.R. China
}

Received November 30, 2018; Accepted July 30, 2019

DOI: $10.3892 /$ ijo.2020.4956

\begin{abstract}
The effective antitumor drug evodiamine (EVO) is attracting increased attention. Therefore, the present study aimed to investigate the effects of EVO on the proliferation, apoptosis and autophagy of human pancreatic cancer (PC) cell lines in vitro and in vivo. Human PANC-1 and SW1990 PC cell lines were treated with different concentrations of EVO and proliferation was detected using a Cell Counting Kit (CCK)-8 assay. Colony formation and wound-healing assays showed that EVO inhibited PC cell viability and migration, and apoptosis was detected using flow cytometry. Western blotting and immunofluorescence detected the expression of proteins in PANC-1 and SW1990 cells. The PANC-1 cells were used to establish an orthotopic pancreatic tumor model in nude mice. Tumor-bearing nude mice were administered with different concentrations of EVO, and growth was monitored. High-resolution positron emission tomography and fluorine-18-labeled fluorodeoxyglucose were used to monitor the tumor/non-tumor (T/NT) ratio and standard uptake value (SUV) of the mice, which were subsequently sacrificed to measure the transplanted tumor weight. Apoptosis increased with increasing EVO concentration. The EVO-treated PC cells exhibited significantly higher expression of LC3II than the controls cells. EVO decreased LC3II, enhanced P62 and inhibited the expression of Akt, extracellular-signal-regulated protein kinase (ERK)1/2 and p38. Compared with the control group, the T/NT ratio, SUV and tumor weight decreased more markedly in the EVO-treated group. The tumor expression of
\end{abstract}

Correspondence to: Dr Hui Chen or Dr Qiyu Zhang, Department of Hepatobiliary and Pancreatic Surgery, The Second Affiliated Hospital and Yuying Children's Hospital, Wenzhou Medical University, 109 West Road, Lucheng, Wenzhou, Zhejiang 325035, P.R. China

E-mail: qiyuz@126.com

E-mail:wzyxych@163.com

Keywords: antitumor, autophagy, apoptosis, immunohistochemistry, nude mouse model, evodiamine phosphorylated AKT, detected using immunohistochemistry, decreased with increasing EVO doses in vivo. EVO induced PC cell apoptosis by inhibiting phosphoinositide 3-kinase/AKT and mitogen-activated protein kinase/ERK and inhibiting the phosphorylation of signal transducer and activator of transcription activator 3 in PC cells to inhibit autophagy, suggesting that EVO may be considered as a novel PC treatment.

\section{Introduction}

Pancreatic cancer (PC) is a malignant tumor that is difficult to diagnose and treat (1). The incidence of PC and its mortality rate have increased significantly in recent years, and with a 5 -year survival rate of $<1 \%$, it has one of the poorest prognoses of malignant tumors (2). PC treatment is mainly based on comprehensive surgical intervention. However, the surgical resection success rate is low and the postoperative recurrence rate is high (3). Chemotherapy is the main form of treatment for unresectable PC and is one of the main strategies to prevent its postoperative recurrence. Gemcitabine is considered the 'gold standard' chemotherapy regimen for advanced PC carcinoma and the postoperative recurrence of PC (4). However, the effective rate of gemcitabine chemotherapy is $<10 \%$, and its long-term use leads to cancer resistance to chemotherapy (5). Therefore, the development of novel and effective chemotherapy drugs is necessary. Studies have shown that curcumin, thymol and other similar compounds significantly inhibit PC cell proliferation $(6,7)$.

Evodiamine (EVO) is a chemical substance extract of plant origin, it is usually extracted from the genus Tetradium, trees of the family Rutaceae (8). EVO has been shown to reduce fat uptake in studies in mice (9). The mechanism underlying the effect of EVO has not been established, however, it is expected that EVO acts primarily as a thermogenic substance (10). It has also reported that EVO can influence serotonin reuptake in the brain (11). In previous years, there have been multiple publications focusing on various aspects of EVO, in terms of its effects on proliferation, apoptosis and autophagy (12-14). As the main component of the Chinese medicine formulation Evodia (15), EVO has inhibitory effects on cervical, lung, colon and nasopharyngeal cancer and other tumor types, likely mediated by the inhibition of tumor proliferation, metastasis 
and angiogenesis and the promotion of tumor cell apoptosis and autophagy (16). The present study was conducted to determine the effects of EVO on the proliferation, apoptosis and autophagy of human PC cell lines and an in situ xenograft model of PC in nude mice, and to investigate its possible mechanism of action.

\section{Materials and methods}

Cell culture, treatment and chemicals. The human SW1990 and PANC-1 PC cell lines were obtained from the Institute of Biochemistry and Cell Biology, Chinese Academy of Sciences (Shanghai, China) and were routinely cultured in Roswell Park Memorial Institute (RPMI)-1640 medium and Dulbecco's modified Eagle's medium (DMEM; both from Gibco; Thermo Fisher Scientific, Inc.). All cell lines were supplemented with $10 \%$ fetal bovine serum (Invitrogen; Thermo Fisher Scientific, Inc.) and $1 \%$ penicillin/streptomycin solution at $37^{\circ} \mathrm{C}$ in a humidified atmosphere of $5 \% \quad \mathrm{CO}_{2}$. The nutrient medium was replaced every 2-3 days and the cells were subcultured when they reached $70-80 \%$ adherence to the bottom of the culture plate, followed by digestion with tryptase. EVO (purity >99\%; Fig. 1A) was purchased from Sigma; Merck KGaA and dissolved in dimethyl sulfoxide (DMSO; Nacalai Tesque, Kyoto, Japan) at $0.2 \mathrm{~mol} / 1$ to produce the stock solution. The final DMSO concentration in the media did not exceed $0.1 \%$. LY294002 (Akt inhibitor), U0126 [extracellular signal-regulated kinase (ERK)1/2 inhibitor] and SB203580 (p38 inhibitor) were obtained from Merck KGaA. Fluorine-18-labeled fluorodeoxyglucose (18F-FDG) was provided by Zhejiang University (Hangzhou, China).

Antibodies. Rabbit monoclonal antibodies against phosphorylated (p)-Akt (Ser473) (D9E) (cat. no. CST 4060), Akt (C67E7; cat. no. CST 4691), p-ERK (Thr202/Tyr204) (D13.14.4E) (cat. no. 4370), ERK (137 F5) (cat. no. 4695), p-p38 (Thr180/Tyr182) (D3F9) (cat. no. 4551), p38 (D13E1) (cat. no. 8690), phosphorylated signal transducer and activator of transcription activator 3 (p-STAT3; Tyr705) (D3A7) (cat. no. 9145), STAT3 (79D7) (cat. no. 4904), P62 (D5E2) (cat. no. 8025) and LC3 (D3U4C) (cat. no. 12741) were purchased from Cell Signaling Technology, Inc. Glyceraldehyde 3-phosphate dehydrogenase (GAPDH; cat. no. sc-47724) and HRP AffiniPure Goat Anti-Rabbit IgG (H+L, cat. no. A32731) were obtained from Santa Cruz Biotechnology, Inc.

Cell survival rate detection using Cell Counting Kit (CCK)-8. The cells were seeded into 96 -well plates at a density of $5 \times 10^{3}$ cells per well in $100 \mu \mathrm{l}$ of the respective medium overnight and were then divided into five groups, each comprising five wells. The cells were treated with different concentrations $(0,0.5,1.0$, 2.5, 5.0 and $10.0 \mu \mathrm{M}$ ) of the EVO at $4^{\circ} \mathrm{C}$, and then cultured the cells at $37^{\circ} \mathrm{C}$ for $48 \mathrm{~h}$. Wells treated with $0.1 \%$ mass fraction dimethyl sulfoxide (DMSO) without the drug were used as the control. The blank retainer group was set at the same time. At $1 \mathrm{~h}$ prior to the end of incubation, $10 \mu \mathrm{l} \mathrm{CCK}-8$ (Beyotime Institute of Biotechnology) was added to each well. The plates were mixed for 10 min on a gyratory shaker, and absorbance at the $450 \mathrm{~nm}$ wavelength was measured with an ELISA reader (BioTek ELx800, BioTek Instruments, Inc.).
Western blot analysis of autophagy-and apoptosis-related protein expression in PC cells. Following treatment, the cells exposed to phosphatase inhibitors were collected, total protein was extracted, the concentration was measured in all samples using the Bradford Protein Assay kit, and the protein samples (30-100 $\mu \mathrm{g}$ ) were separated using 10-15\% sodium dodecyl sulfate-polyacrylamide gel electrophoresis. The separated proteins were then transferred onto a polyvinylidene fluoride membrane, which was blocked for $1.5 \mathrm{~h}$ in blocking buffer consisting of $5 \%$ milk in Tris-buffered saline (TBS) containing $0.05 \%$ Tween-20, and then incubated with the different primary antibodies (1:400) overnight at $4^{\circ} \mathrm{C}$. The membrane was washed with TBS containing $0.1 \%$ Tween-20, incubated with a horseradish peroxidase (HRP)-conjugated secondary antibody $(1: 1,000)$ for $1 \mathrm{~h}$ at $25^{\circ} \mathrm{C}$. Primary and secondary antibodies were diluted in Tris-buffered saline (TBS) containing $0.05 \%$ Tween-20. The immunoreactive bands were then visualized using enhanced chemiluminescence kits. The density of the immunoreactive bands was analyzed using the Image $\mathbf{J}$ software (v1.8.0; National Institute of Health).

Scratch wound model. The PC cells $\left(5 \times 10^{5}\right.$ cells/well) were seeded in 6-well plates and allowed to adhere overnight without serum-starvation. At $80-90 \%$ confluence, a 'reference line' scratch was made at the bottom of the plate using a sterile $100-\mu 1$ pipette tip. Following washing three times with phosphate-buffered saline (PBS), the cells were incubated with $\operatorname{EVO}(1,5$ and $10 \mu \mathrm{M})$ or the vehicle (DMSO) medium to detect cells in the absence of cell growth migration. Following processing for 0 and $24 \mathrm{~h}$, a digital camera following analysis with an MX4R Inspection microscope (Canon, Inc., magnification, x100) was used to capture micrographs of the cells that had migrated across the reference line in different fields. Compared with the control, the rate of movement was quantified according to the migrated distance of the cells from the reference line to the center. All experiments were repeated three times.

Cell colony-forming assay. To determine the cell colony-forming units, 500 cells per well were seeded into 6 -well plates with $2 \mathrm{ml}$ of culture medium overnight. The cells were pretreated with EVO, mixed with DMSO for 8-12 h, washed twice with PBS, and then transferred to fresh medium to allow the cells to grow for 7 days. The colonies were washed with PBS and then fixed with $4 \%$ methanol for $15 \mathrm{~min}$ at room temperature. The cells were washed then twice with PBS and stained with $1 \%$ crystal violet ( $25 \%$ methanol) for $10 \mathrm{~min}$ at room temperature. An image of the stained cell colonies was captured using a digital camera following analysis with an MX4R Inspection microscope. The cell size in the field of view is magnified 10 times. Three independent experiments were conducted for each assay.

Assessment of apoptosis using flow cytometry. Apoptosis was assessed using an Annexin V/propidium iodide (PI) kit (BD Biosciences Pharmingen). Exponentially growing cells were seeded in 6-well plates and then treated with EVO (1,5 and $10 \mu \mathrm{M}$ ) or DMSO (vehicle control, final concentration of $0.1 \%$ ) for $48 \mathrm{~h}$. Treated cells were harvested at room temperature and stained with Annexin V and PI for $15 \mathrm{~min}$ and 
A

Evodiamine

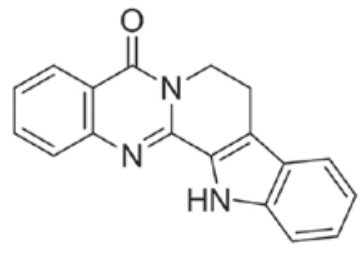

B

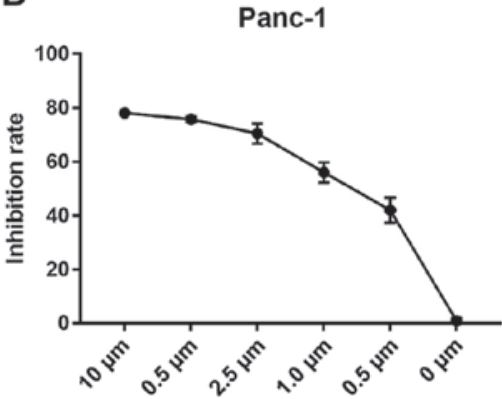

C

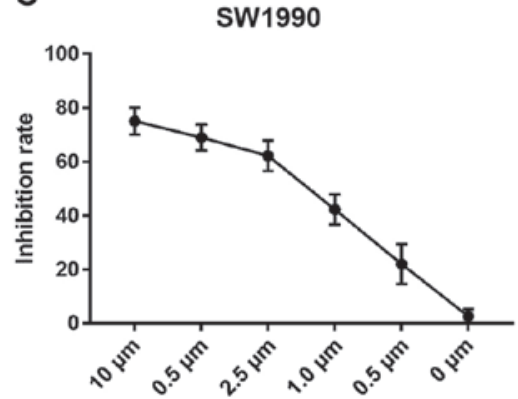

Figure 1. Cell growth effects of EVO on PC cells. (A) Chemical structure of EVO. Graphs show the cell growth of (B) PANC-1 and (C) SW1990 PC cell lines treated with EVO at different concentrations for $48 \mathrm{~h}$. Cell viability was determined using a Cell Counting Kit- 8 assay. Data were obtained from three independent experiments performed in triplicate. EVO, evodiamine; PC, pancreatic cancer.

analyzed using flow cytometry with a FACSCalibur instrument (BD Biosciences). The exported data was processed and analyzed using FlowJo Software version 7.6.1 (FlowJo LLC).

Immunofluorescence analysis of LC3 expression in PC cells. The PC cells were cultured in a nutrient-deficient medium for $24 \mathrm{~h}$ (17). The well-attached cells $\left(5 \times 10^{5}\right.$ cells/well) were placed directly onto coverslips to allow them to grow. The coverslips containing logarithmic growth-phase cells were placed in a 6 -well plate, fixed in $4 \%$ paraformaldehyde $(1 \mathrm{ml}$ per well) for $30 \mathrm{~min}$ and washed with PBS three times for 5 min each. The cells were then permeabilized with PBS containing 1\% Triton X-100 (PBST) for $15 \mathrm{~min}$, washed three times with PBS for 5 min each, and blocked with blocking solution (normal donkey serum; Gibco; Thermo Fisher Scientific, Inc.) for $30 \mathrm{~min}$ in $4^{\circ} \mathrm{C}$. Non-specific staining was assessed by replacing the LC3 antibody (D3U4C, cat. no. 12741) with PBS. In the control group, only DMSO was added. The coverslips were subsequently incubated with anti-LC3 (1:400) overnight at $4^{\circ} \mathrm{C}$ and rinsed with PBST three times (flushing for $5 \mathrm{~min}$ each time). For indirect immunofluorescence, the coverslips were further incubated with HRP AffiniPure Goat Anti-Rabbit IgG $(\mathrm{H}+\mathrm{L}$, cat. no. A32731; 1:50) in the dark at room temperature for $1 \mathrm{~h}$ and then rinsed with PBST three times. Primary and secondary antibodies were diluted in Tris-buffered saline (TBS) containing $0.05 \%$ Tween-20. Following each 5-min rinse, the coverslips were rinsed again with distilled water, one drop of the encapsulated tablet was dropped, the coverslips were mounted and then examined using fluorescence microscopy.

4',6-diamidino-2-phenylindole (DAPI) staining. Following the indicated treatments, the cells were fixed with $4 \%$ paraformaldehyde for $20 \mathrm{~min}$ and were washed thoroughly with PBS between each reaction to remove any residual solvent. The cells were stained with DAPI for $15 \mathrm{~min}$ in $4^{\circ} \mathrm{C}$ and viewed under a fluorescence microscope equipped with an ultraviolet (UV) filter.

Orthotopic PC xenograft nude mouse model establishment. Female BALB/c nude mice, (age, 4-6 weeks; weight, weight 20-25 g) were obtained from Shanghai SLAC Laboratory Animal Co., Ltd. for tumor implantation. All mice were maintained in a sterile environment under constant temperature $\left(25^{\circ} \mathrm{C}\right)$ and humidity, with a 12 -h light/dark cycle, and free access to food and water. Their care adhered to the laboratory animal regulations of the Ministry of Science and Technology of the People's Republic of China (http://www.most.gov. cn/kytj/kytjzcwj/200411/t20041108_32465.hgrowthtm). The feed and drinking water of nude mice were purchased from Beijing Keao Xieli Feed Co., Ltd.

A total of 40 mice were used, and the food, water and bedding of the immunocompromised mice were sterilized and replaced at least once per week. PANC-1 PC cells $\left(5 \times 10^{6}\right)$ in $\log$ phase were collected in $50 \mu \mathrm{l}$ serum-free DMEM per mouse and orthotopically implanted into anesthetized athymic nude mice. In brief, a small laparotomy was performed on the left upper quadrant of each mouse. Anesthesia of the mice was induced with $3 \%$ isoflurane and maintained with $2 \%$ isoflurane. The PANC-1 cells were injected subcapsularly in a region of the pancreas just beneath the spleen. Successful subcapsular intra-pancreatic injection of the cancer cells was identified by the appearance of a fluid bleb without intraperitoneal (i.p) leakage (18). All surgical procedures were performed under sterile conditions using a 10X microscope. At 1 week post-implantation, the mice were medially randomized into four groups to receive i.p. injections of the vehicle $(0.9 \%$ sodium chloride) or different concentrations of EVO (10, 20 and $30 \mathrm{mg} / \mathrm{kg}$ ) (19) three times per week for 2 weeks.

Micro positron emission tomography (PET) imaging. PET imaging was performed 4 weeks after the nude mice were treated. The mice were fasted 1 day before imaging and injected via the tail vein with $0.1 \mathrm{mCi} 18 \mathrm{~F}-\mathrm{FDG} / \mathrm{mouse}$. Anesthesia of the mice was induced using $3 \%$ isoflurane and maintained with $2 \%$ isoflurane, placed in the prone position, and scanned along the long axis of the Micro PET scanner. Data were collected following the administration of $18 \mathrm{~F}-\mathrm{FDG}-\mathrm{PET}$ for $10 \mathrm{~min}$ and the ingestion time was $1 \mathrm{~h}$ after tracer injection. Static acquisition in 3D was performed using a micro PET imaging system (R4, Concorde Microsystems, Inc.). For quantitation, the regions of interest (ROIs) method and standard uptake values (SUVs) were used and statistically analyzed using micro PET ASIPro6.0.5.0 (Concorde Microsystems, Inc.). The ROI perimeters were drawn around the tumor and the area around the coronal section showing the best tumor depiction. All images were displayed using the same color scale. The highest and average intakes (percentage injected doses) in 
A

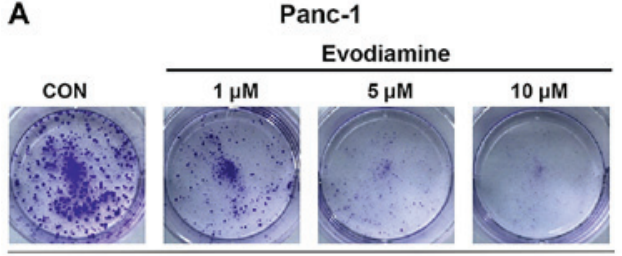

B

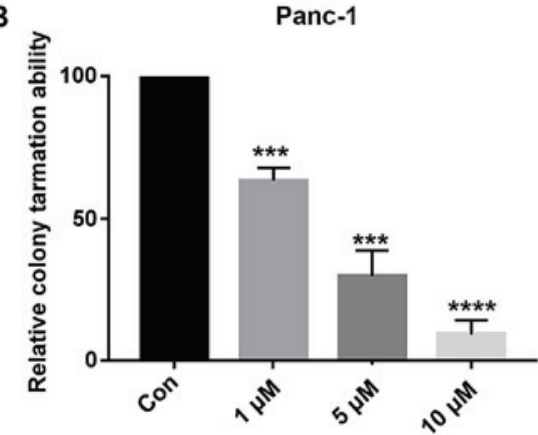

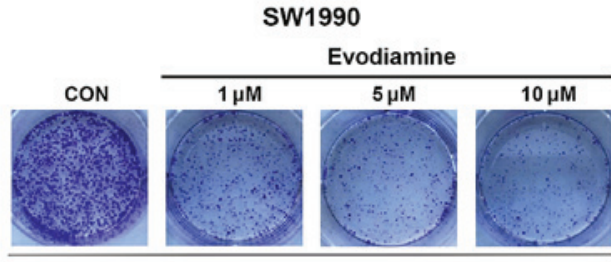

SW1990

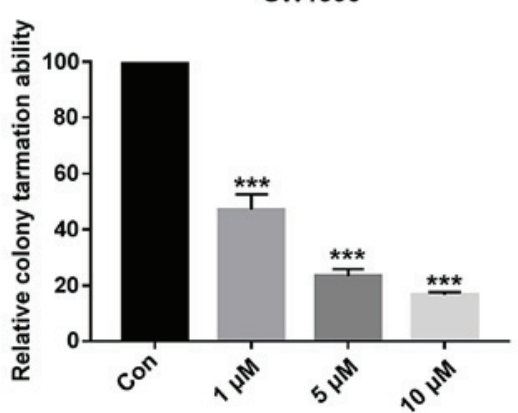

Figure 2. EVO inhibits colony formation in pancreatic cancer cells. (A) PANC-1 and SW1990 cells were exposed to different EVO concentrations (1, 5 and $10 \mu \mathrm{M})$ and examined using a clonogenic assay. Dimethyl sulfoxide $(1 \mu \mathrm{l})$ was added as a negative control. Data were obtained from three independent experiments performed in triplicate. (B) Results of the relative colony formation abilities of PANC-1 and SW1990 cells were calculated. ${ }^{* * *}$ P $<0.001$ and ${ }^{* * * * *} \mathrm{P}<0.0001$ vs. Con. EVO, evodiamine; Con, control.

the tumor and surrounding ROIs, respectively were recorded and calculated (20). The tumor/non-tumor $(\mathrm{T} / \mathrm{N})$ ratio, which indicates tumor growth metabolism, of the semi-quantitative analysis was calculated using the following formula: $\mathrm{T} / \mathrm{N}$ ratio $=$ maximum pixel uptake in the tumor ROI / average pixel uptake in the surrounding ROI. The SUVs for ROIs of different groups were calculated as follows: $\mathrm{SUV}=$ radioactivity concentration in $\mathrm{ROI}(\mathrm{mCi} / \mathrm{mL}) \mathrm{x}$ body weight $(\mathrm{g}) /$ injection dose $(\mathrm{mCi})$, and compared. At the end of the experiment, the mice were sacrificed by cervical dislocation 1 week after the final PET imaging. The tumor samples were weighed and fresh frozen in liquid nitrogen and $10 \%$ formalin prior to analysis. The mice were sacrificed 8 weeks after implantation.

Immunohistochemical analysis of related proteins $p-A K T$, $p$-ERK and $p-P 38$. The formalin-fixed paraffin-embedded tumor tissue samples were sectioned, deparaffinized, rehydrated, blocked with $10 \%$ goat serum (Gibco; Thermo Fisher Scientific, Inc.) in PBS for $1 \mathrm{~h}\left(4^{\circ} \mathrm{C}\right)$, and then immunostained. The sections $(25 \times 75 \mathrm{~mm})$ were incubated with p-Akt (Ser473, D9E, cat. no. CST 4060), p-ERK (Thr202/Tyr204) (D13.14.4E, cat. no. 4370) and p-p38 (Thr180/Tyr182, D3F9, cat. no. 4551) in a moist chamber overnight at $4^{\circ} \mathrm{C}$. Following washing with PBS, the sections were incubated with an HRP-conjugated secondary antibody for an additional $30 \mathrm{~min}$ at room temperature. Following staining with hematoxylin, the sections were evaluated using a microscope (Olympus BX51, Olympus Corporation). Non-specific primary antibody staining was assessed by substituting PBS for the primary antibody, and at least 10 fields were randomly selected from each section for evaluation. The images were analyzed using Image-Pro Plus 6.0 software (Media Cybernetics, Inc.).

Statistical analysis. The data are presented as the mean \pm standard error of the mean and the experiments were repeated three times $(n=3)$. The statistical package for the social sciences
(SPSS) 13.0 software (SPSS, Inc.) was used for statistical analysis. Differences in mean values between the groups were initially analyzed using one-way analysis of variance.

\section{Results}

Cell growth effects of EVO in PC cells. The inhibitory effects of EVO on PANC-1 and SW1990 PC cell lines were evaluated following incubation with different concentrations for $48 \mathrm{~h}$. As shown in Fig. 1B and C, EVO dose-dependently reduced the viability of SW1990 and PANC-1 cells, indicating that EVO markedly inhibited the growth of PC cells.

EVO inhibits colony formation in PC cells. The colony-forming ability characterizes the independent survival and environmental adaptation of cancer cells. Therefore, the effect of EVO on the clonogenic survival of PC cells was evaluated using a colony formation assay in plates. As shown in Fig. 2A and B, EVO dose-dependently inhibited the clonogenic survival of PANC-1and SW1990 cells after 7 days of treatment. These findings indicated that EVO potently inhibited PC cell growth in vitro and may be useful for the treatment of PC.

EVO induces apoptosis of PC cells. The role of apoptosis in EVO-induced cell death was then evaluated using Annexin V-fluorescein isothiocyanate and PI staining. The PANC-1 and SW1990 cells were treated with EVO (1.0, 5.0 and $10 \mu \mathrm{M}$ ) for $48 \mathrm{~h}$ and then stained with Annexin V and PI for the analysis of apoptosis using flow cytometry. As shown in Fig. 3A and B, the exposure of cells to EVO at various concentrations for $48 \mathrm{~h}$ led to a dose-dependent increase in apoptosis. To determine whether EVO-induced cell death was related to apoptosis, DAPI staining was performed to analyze the changes in nuclear morphology. The results revealed condensed and fragmented nuclei in both cell types following $24 \mathrm{~h}$ of EVO $(10 \mu \mathrm{M})$ treatment (Fig. 3C). 
A
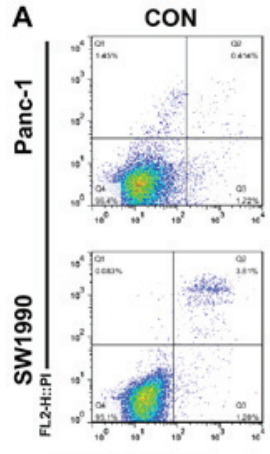

$1.0 \mu \mathrm{M}$
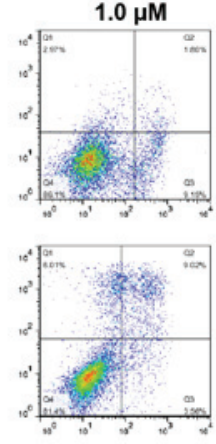
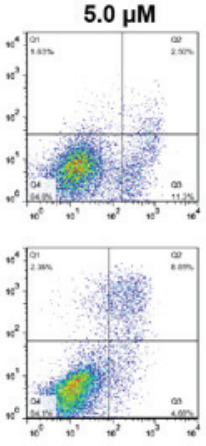
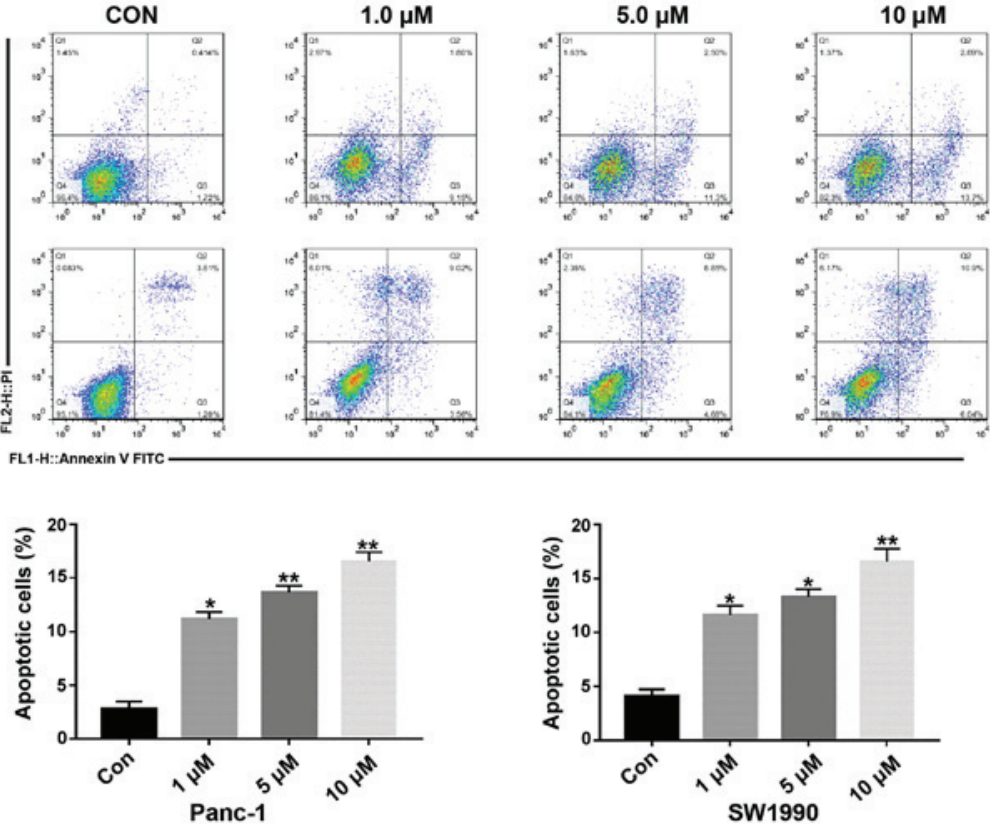

SW1990
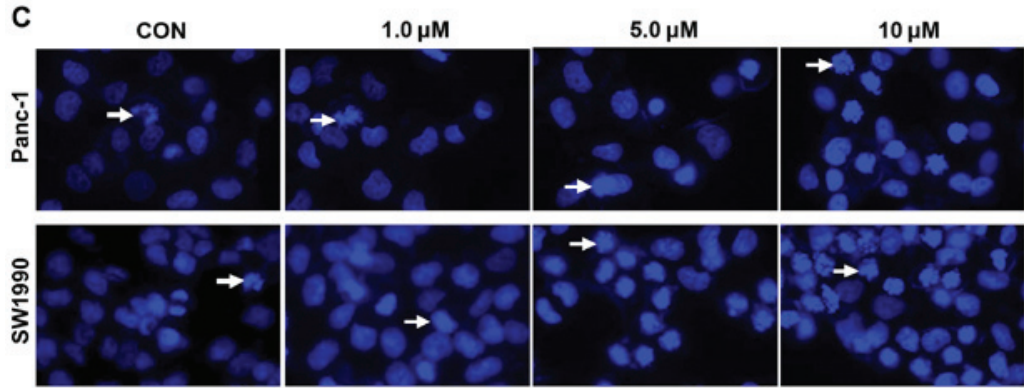

Figure 3. EVO induces the apoptosis of pancreatic cancer cells. (A) EVO induced the apoptosis of PANC-1 and SW1990 cells. PANC-1 and SW1990 cells were treated with EVO at the indicated concentrations $(1,5$ and $10 \mu \mathrm{M})$ or dimethyl sulfoxide for $48 \mathrm{~h}$ and then stained with Annexin V and PI, followed by detection using flow cytometry. Representative plots are shown. (B) Apoptosis results from PANC-1 and SW1990 cells were calculated. * $<<0.05$ and ${ }^{* *} \mathrm{P}<0.01$ vs. Con. (C) Cells were treated with different concentrations of EVO $(0-10 \mu \mathrm{M})$ for $24 \mathrm{~h}$ and then subjected to DAPI staining (blue) and analyzed by fluorescence microscopy. The arrows indicate apoptotic cells. Magnification, x100. EVO, evodiamine; Con, control; PI, propidium iodide.

EVO inhibits the expression of apoptosis-related proteins in PC cells. Previous data showed that the AKT/PI3K signaling pathway serves an important role in the occurrence and development of various tumor cells (19). Therefore, the present study performed western blot analysis of PC cells to examine whether EVO inhibits the expression of AKT, ERK1/2 and P38. As shown in Fig. 4A and B, EVO inhibited the phosphorylation of AKT, ERK1/2 and P38 in a dose-dependent manner. The role of $\mathrm{p} 38, \mathrm{AkT}$ and erk1/2 in EVO-induced cell viability was also investigated. PANC-1 and SW1990 cells pretreated with LY294002, SB203580 or U0126 exhibited decreased cell viability compared with those treated only with EVO (Fig. 4C).

EVO inhibits the expression of autophagy-related LC3 and regulates apoptosis-related protein expression in PC cells. Treatment with EVO significantly reduced the levels of apoptotic marker STAT3 in PC cells (Fig. 5A and B). In addition, EVO reduced the expression of LC3-II and enhanced that of P62, autophagy markers in both cell lines, indicating that autophagy was inhibited (Fig. 5A and B). The distribution of LC3 was observed using cellular immunostaining (Fig. 5C). Treatment with EVO decreased the number of LC3 puncta, also indicating that autophagy was inhibited (Fig. 5C and D).
EVO decreases $P C$ cell migration. To evaluate the inhibitory effects of EVO on cell migration, a scratch wound model was used. The wound areas were marked and images were captured at 0 and $24 \mathrm{~h}$. Compared with the control PANC-1 and SW1990 cells, those treated with EVO at different concentrations (1.0, 5.0 and $10 \mu \mathrm{M})$ migrated into the wound with a significantly decreased distance between the edges after $24 \mathrm{~h}$, as shown in Fig. 6A and B.

Micro PET imaging. The survival rate was of the mice was $100 \%$ and all tumors were observed in the coronal plane of the micro PET. The arrows in Fig. 7A show the tumor localization. It was found that the drug decreased the T/NT ratio in a concentration-dependent manner. The T/NT ratios of the EVO 10, 20 and $30 \mathrm{mg} / \mathrm{kg}$ groups $(2.95 \pm 0.090,1.83 \pm 0.260$ and $1.31 \pm 0.142$, respectively) were lower than those of the control group (0.9\% sodium chloride, $3.52 \pm 0.095, \mathrm{P}<0.05$, Fig. 7B). The SUVs of the control and the 10, 20 and $30 \mathrm{mg} / \mathrm{kg}$ EVO groups were $5.21 \pm 0.33,4.21 \pm 0.16,3.11 \pm 0.26$ and $2.36 \pm 0.35$, respectively (Fig. 7C). The regional average intake of the tracer was downregulated in an EVO concentration-dependent manner compared with that of the in vivo control group $(\mathrm{P}<0.05)$. 
A

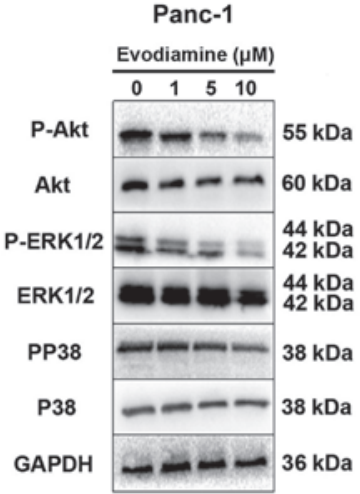

B
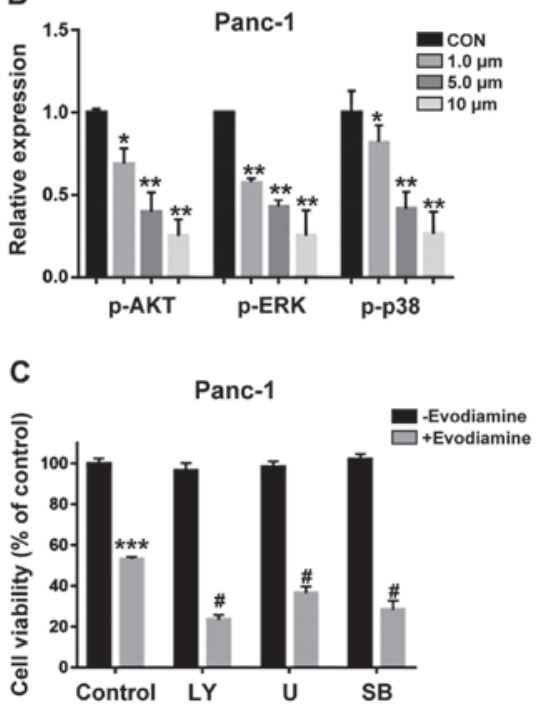

SW1990

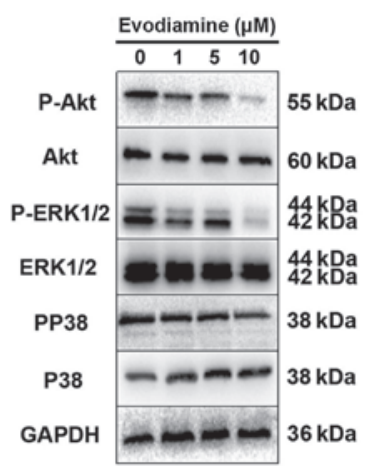

SW1990
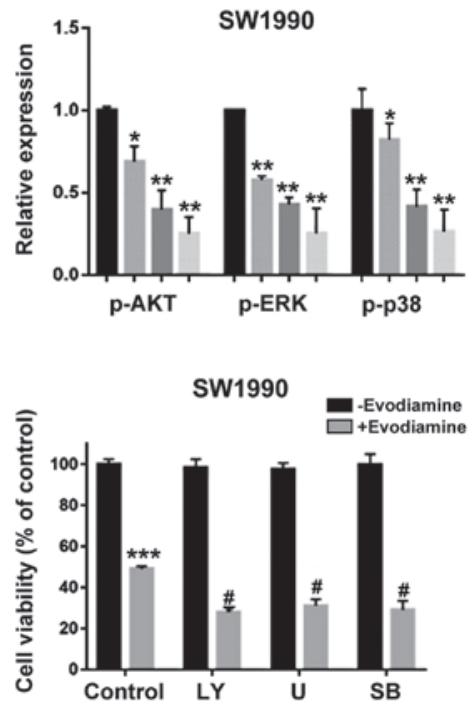

Figure 4. EVO inhibits anti-apoptotic signal proteins through inhibition of Akt, ERK1/2, and p38 in PANC-1 and SW1990 cells. (A) PANC-1 and SW1990 cells were treated with different concentrations of EVO $(0-10 \mu \mathrm{M})$ for $24 \mathrm{~h}$ and then subjected to western blotting. The levels of EVO of Akt, ERK1/2 and p38 were investigated by western blotting with GAPDH as an internal control. (B) Quantitative results of the protein levels of Akt, ERK1/2 and p38, which were adjusted with the GAPDH protein level. (C) PANC-1 and SW1990 cells were pretreated with LY294002 (20 $\mu \mathrm{M})$, SB203580 (20 $\mu \mathrm{M})$ or U0126 (10 $\mu \mathrm{M})$ for $1 \mathrm{~h}$ followed by treatment with EVO $(2 \mu \mathrm{M})$ for $24 \mathrm{~h}$ and analyzed using a Cell Counting Kit- 8 assay. Results are shown as the mean $\pm \mathrm{SD}$. " $\mathrm{P}<0.05$, ${ }^{* * *} \mathrm{P}<0.01$ and ${ }^{* * *} \mathrm{P}<0.001$ vs. control group; ${ }^{*} \mathrm{P}<0.05$ vs. evodiamine alone group. EVO, evodiamine; ERK, extracellular signal-regulated kinase; P-, phosphorylated; GAPDH, glyceraldehyde 3-phosphate dehydrogenase; CON, control; LY, LY294002; U, U0126; SB, SB203580.

EVO inhibits orthotopic xenograft growth in nude mice. The effects of EVO on orthotopic xenografts in nude mice were investigated (Fig. 8A). The tumor weights (Fig. 8B and C) of the EVO 10,20 and $30 \mathrm{mg} / \mathrm{kg}$ groups, were $0.82 \pm 0.13$, $0.67 \pm 0.18$ and $0.23 \pm 0.17 \mathrm{~g}$, respectively, compared with that of the control group $(1.58 \pm 0.27 \mathrm{~g})$. As the concentration of EVO increased, the body weight of nude mice also increased. In addition, the volume of tumors in the nude mice decreased with increasing drug concentration (Fig. 8D). These results showed that EVO inhibited tumor growth in the nude mice in a concentration-dependent manner.

Immunohistochemistry of the expression of $p-A K T, p-E R K$ and p-P38 in tumor tissues. The detection of p-AKT, p-ERK and p-P38 indicated the inhibition of PC cell proliferation in the treatment group (Fig. 9A and B). The expression levels of p-AKT, p-ERK, and p-P38 were microscopically examined at $\mathrm{x} 400$ magnification. Compared with those in the control group, the expression levels of p-AKT, p-ERK and p-P38 in the tumor tissues decreased significantly in a concentration-dependent manner (Fig. 9B).

\section{Discussion}

$\mathrm{PC}$, which is one of the most malignant types of cancer worldwide (5), has a high mortality rate and usually progresses to an untreatable state (21). Gemcitabine is used to treat patients with PC, but its effectiveness is $<12 \%$ and it is associated with considerable toxicity (2). Therefore, novel therapeutic strategies require investigation to improve treatment outcomes. EVO, a quinolone alkaloid, is traditionally used for stomachache and headache in China. The present study investigated the effects and precise mechanism of action of EVO in PC and found that EVO significantly increased apoptosis in a concentration-dependent manner by increasing apoptotic bodies and inducing chromosome condensation, which serves an effective role in PC inhibition.

Early reports indicate that the treatment of A375-S2 human melanoma cells with EVO negatively affected the PI3K/AKT signaling pathway $(22,23)$, Studies have shown that EVO significantly inhibits the proliferation of SGC-7901 cells and induces cell cycle arrest in the G2/M phase, indicating that autophagy and apoptosis were activated during EVO-induced SGC-7901 
A

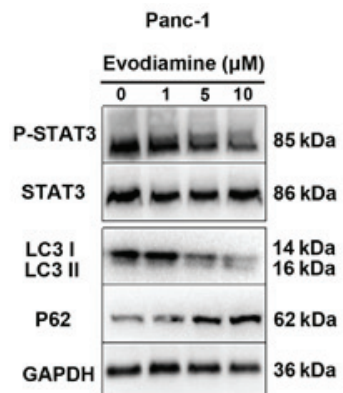

B

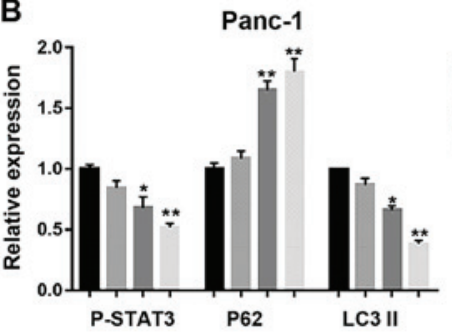

SW1990

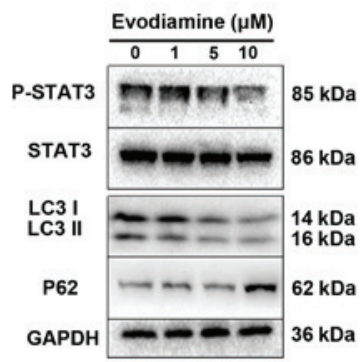

SW1990

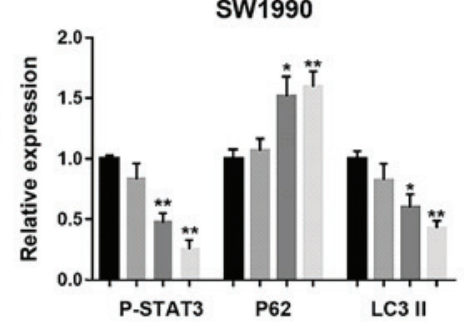

$5.0 \mu \mathrm{M}$
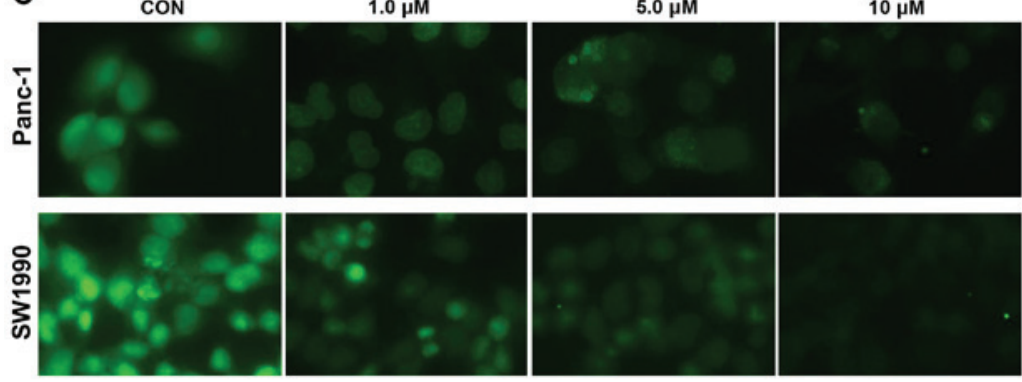

SW1990
D

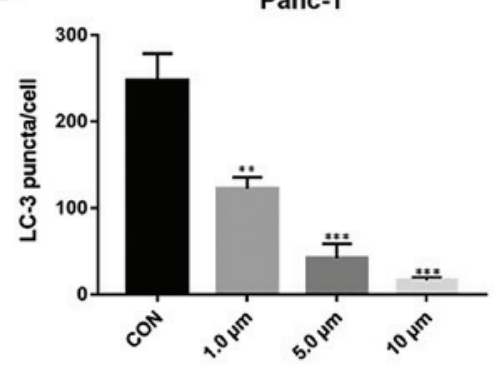

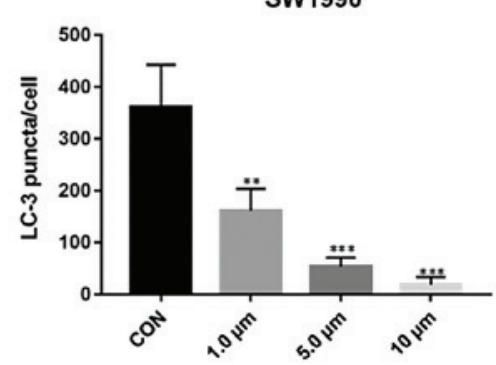

Figure 5. EVO inhibits the expression of autophagy-related LC3 and regulates apoptosis-related protein expression in PC cells. (A) Treatment with EVO induced progressive inhibition of autophagy in PC cell lines. Following culture in a nutrient-deficient medium for $24 \mathrm{~h}, \mathrm{PANC}-1$ and SW1990 cells were treated with different concentration of EVO $(0,1.0,5.0$ and $10 \mu \mathrm{m})$ and harvested at the indicated incubation times. Expression levels of LC3, STAT3 and GAPDH were determined by western blot analysis. (B) Densitometry analysis of the western blotting results. (C) PANC-1 and SW1990 cells were immunostained with anti-LC3 (green). Insets indicate magnified images of the boxed area. Magnification, x100. (D) At least 200 cells on each slide were counted and the percentages of LC3 puncta-containing cells were calculated. Data shown are presented as the mean $\pm \mathrm{SD}$ of three individual experiments. ${ }^{*} \mathrm{P}<0.05$, $^{* *} \mathrm{P}<0.01$ and ${ }^{* * *} \mathrm{P}<0.001$ vs. CON. PC, pancreatic cancer; EVO, evodiamine; STAT3, signal transducer and activator of transcription activator 3; P-, phosphorylated; GAPDH, glyceraldehyde 3-phosphate dehydrogenase; CON, control.

cell death (24). EVO also affected the cell cycle of U87-MG astrocytes (25). In the present study, different concentrations $(0,1$, 5 and $10 \mu \mathrm{m})$ of EVO were used to treat PANC-1 and SW1990 PC cell lines in vitro. It was found that PC cell viability and apoptosis were increased by inhibiting the PI3K/AKT pathway (26). The results showed that increasing the EVO concentration between 1 and $10 \mu \mathrm{M}$ inhibited the growth of tumor cells significantly and their apoptosis became more obvious.

In the present study, EVO exhibited dose-dependent cytotoxicity with a half-maximal inhibitory concentration of $\sim 2 \mu \mathrm{M}$, and the results were similar in the two cell lines. Combined with the results of the CCK assay and the pre-experiment results, it was found that the effect of EVO on PC was concentration-dependent in the $0-10-\mu \mathrm{m}$ range, but the change was not obvious $>10 \mu \mathrm{m}$, thus the experiment was suspended at these concentrations. It was found that, in $72 \mathrm{~h}, \mathrm{PC}$ cell death occurred instead of apoptosis, therefore the duration of $48 \mathrm{~h}$ was used. The experiments demonstrated that AKT was expressed in PC cells. It is reported that PC growth can be suppressed by inhibiting the expression of AKT (19). The present study analyzed protein expression using western blotting, which indicated that EVO downregulated the expression of $\mathrm{p}-\mathrm{AKT}$, 


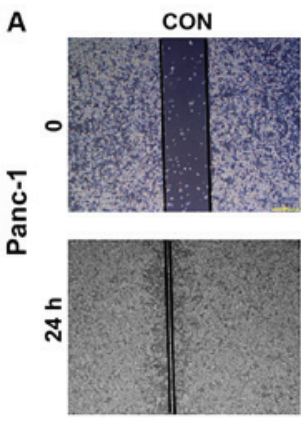

CON

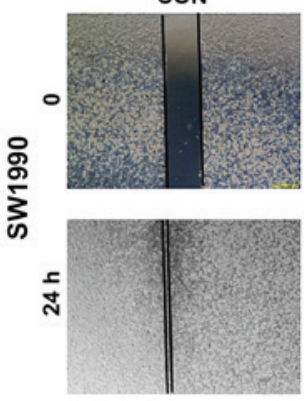

B

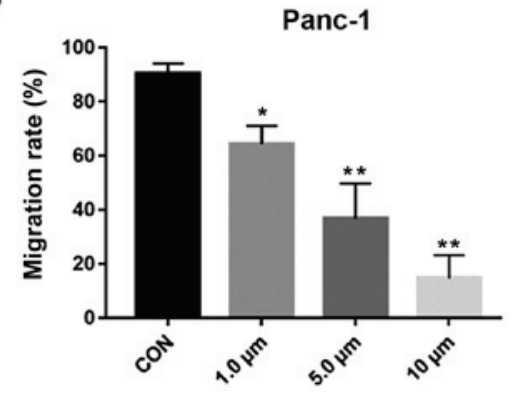

$1.0 \mu \mathrm{M}$

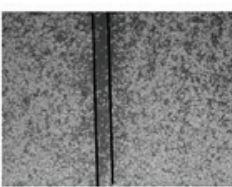

$1.0 \mu \mathrm{M}$
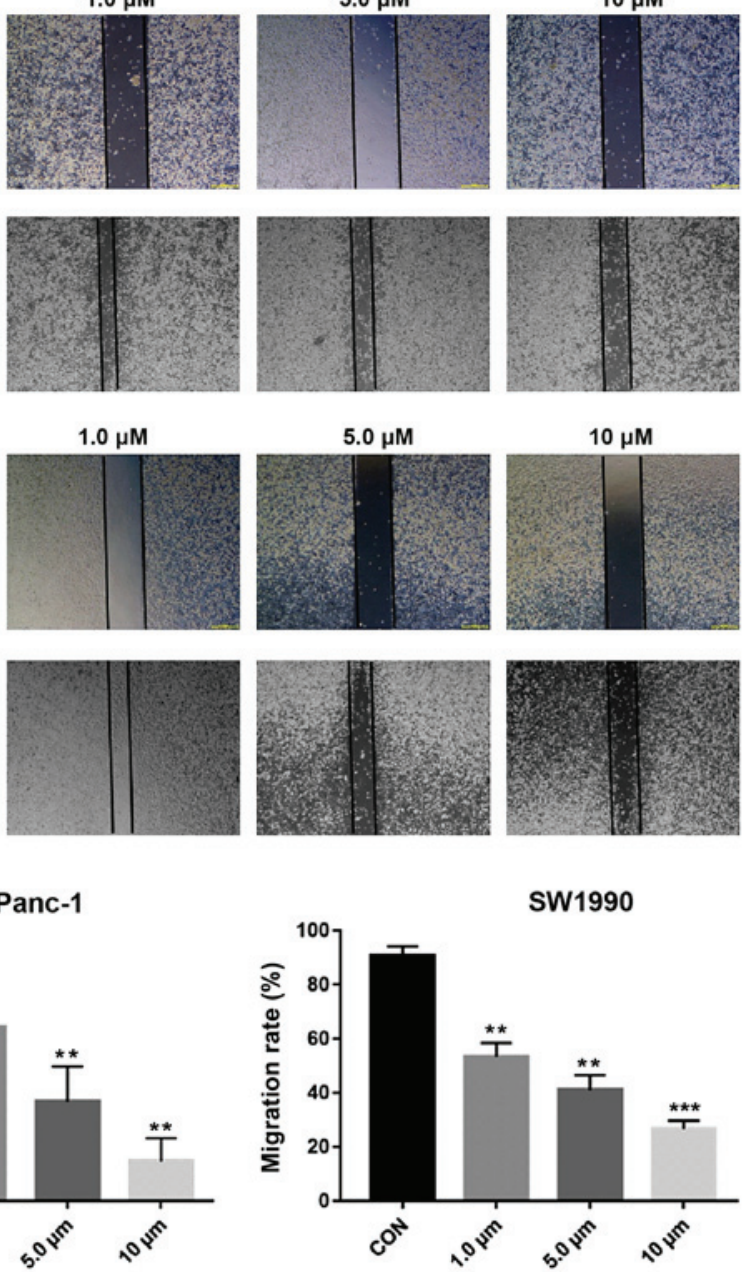

Figure 6. EVO reduces cell migration in pancreatic cancer cells. (A) PANC-1 and SW1990 cells were grown into a single layer, then a wound-healing assay for migration was performed by scraping the cells with a yellow tip. The cells were treated with different concentrations $(1,5$ and $10 \mu \mathrm{M})$ of EVO and allowed to migrate to the scratched area for $24 \mathrm{~h}$. The data obtained is from three independent experiments. Magnification, x100. (B) Results of wound healing assays from PANC-1 and SW1990 were calculated. ${ }^{* *} \mathrm{P}<0.01,{ }^{* * *} \mathrm{P}<0.001$ vs. CON. EVO, evodiamine; CON, control.

A

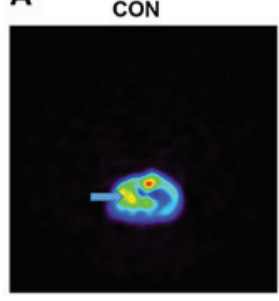

B

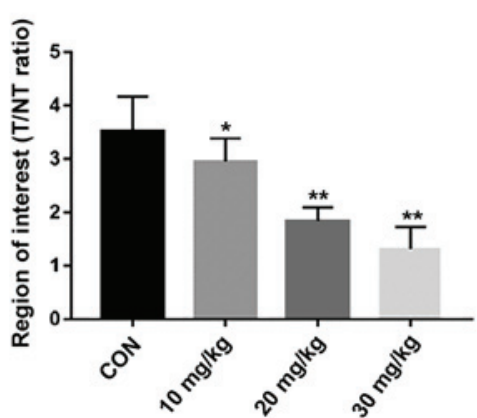

$20 \mathrm{mg} / \mathrm{Kg}$
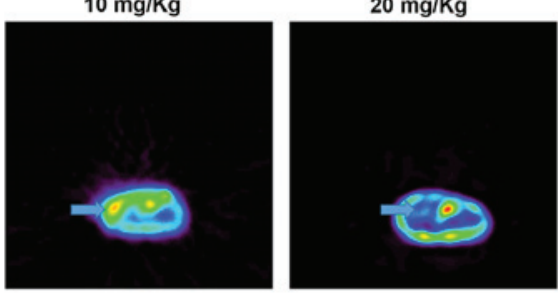

C

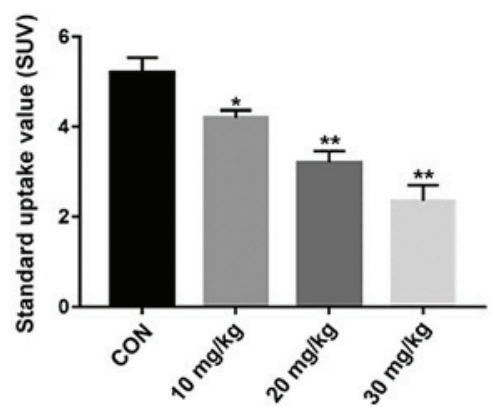

Figure 7. PANC-1 cells were used to establish an orthotopic pancreatic cancer xenograft animal model. (A) Mice bearing orthotopically implanted tumors were imaged by Micro PET for fluorine-18-labeled fluorodeoxyglucose uptake 4 weeks after drug treatment was completed. Micro PET showed transverse sections of orthotopic xenografts in nude mice. The arrow indicates the location of the tumor. The (B) T/NT ratio and (C) SUVs were lower than those in the control group with increasing EVO concentrations. ${ }^{*} \mathrm{P}<0.05$ vs. CON; ${ }^{* *} \mathrm{P}<0.01$ vs. CON. EVO, evodiamine; CON, control; T/NT, tumor/non-tumor; Micro PET, micro positron emission tomography. 

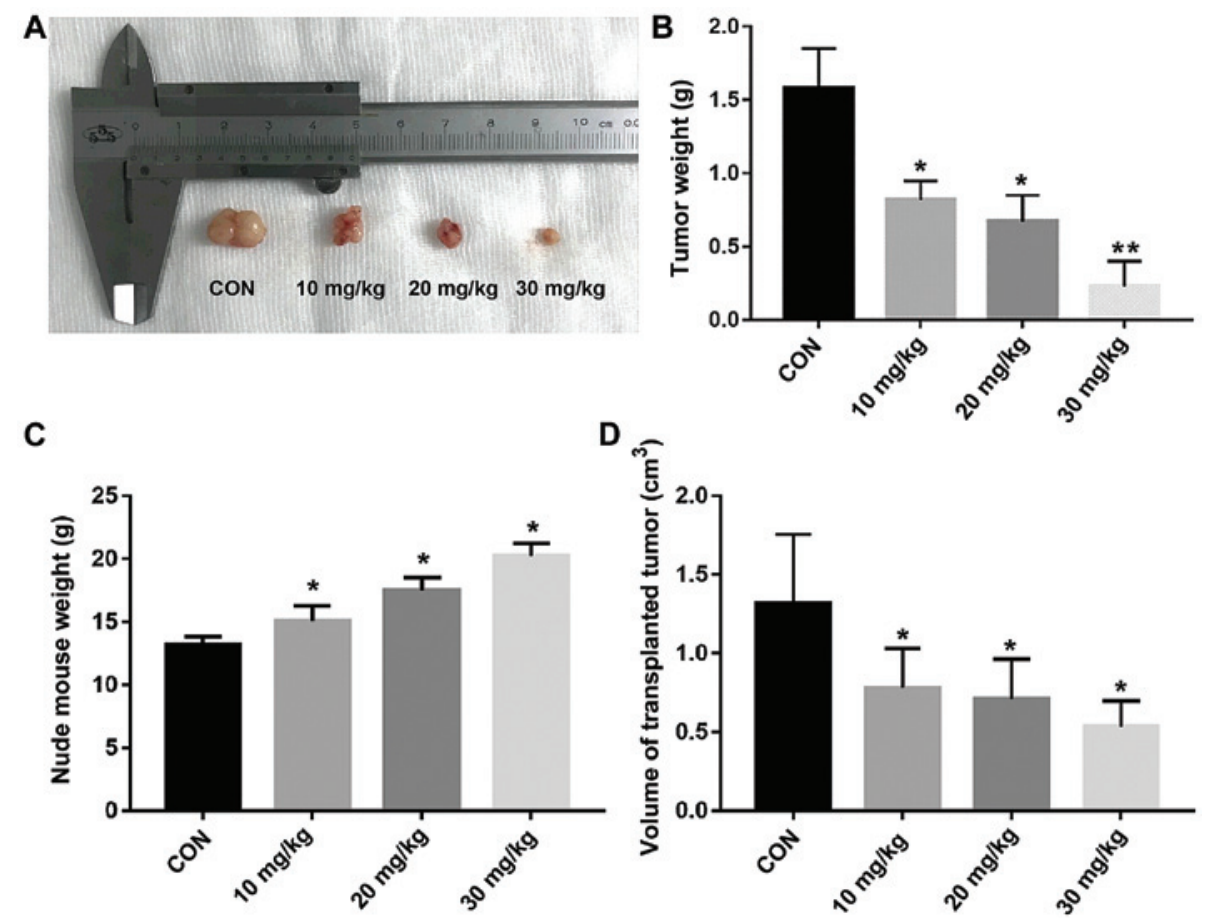

Figure 8. Orthotopic xenograft growth. (A) Representative photographs of the xenograft tumors. (B) The weights of the orthotopic xenograft tumors were examined following sacrifice of the mice. (C) Total body weight of the mice. (D) Volumes of the xenograft tumors. " $\mathrm{P}<0.05$ vs. CON; ${ }^{* *} \mathrm{P}<0.01$ vs. CON. CON, control.
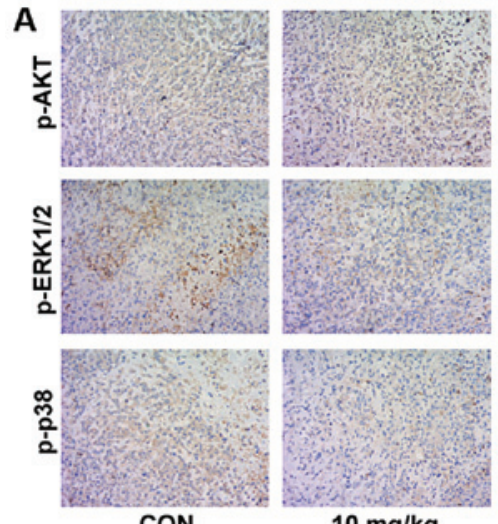

CON

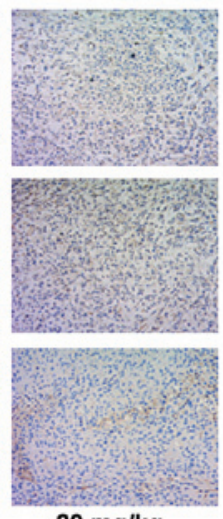

$20 \mathrm{mg} / \mathrm{kg}$
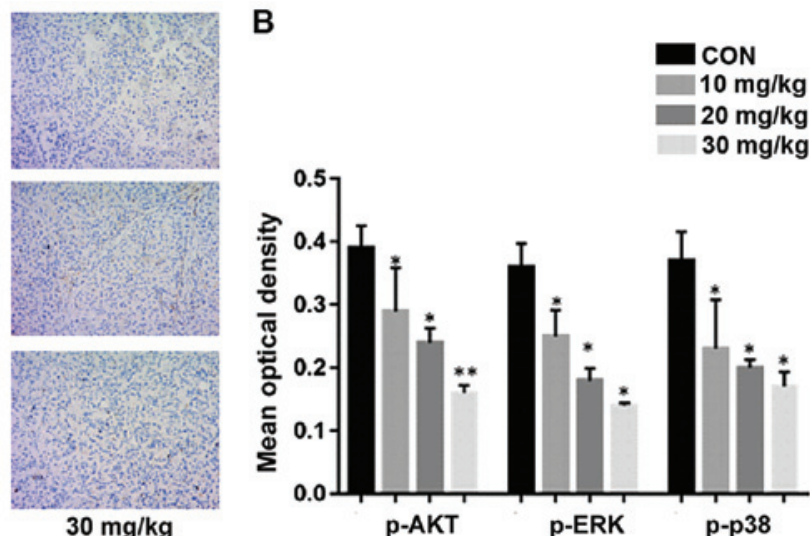

Figure 9. Expression of p-AKT,p-ERK and p-P38 in xenograft tumor tissues. (A) Expression of p-AKT,p-ERK1/2 and p-P38 as detected by immunohistochemistry (magnification, x200). (B) Mean optical density of p-AKT, p-ERK1/2 and p-P38 in orthotopic transplanted tumors. ${ }^{*} \mathrm{P}<0.05$ vs. CON; ${ }^{* *} \mathrm{P}<0.01$ vs. CON. CON, control; ERK, extracellular signal-regulated kinase; p-, phosphorylated.

p-ERK and, p-P38 in PC cells in a concentration-dependent manner. Multiple protein kinases, such as the PI3K/AKT and mitogen-activated protein kinase (MAPK)/ERK pathways, in cells are involved in cell survival and proliferation (27). MAPKs have been shown to be involved in the regulation of apoptosis and cell cycle-related signaling pathways in a variety of cell models. MAPKs consists of three major members, ERK, c-Jun N-terminal kinase and P38 MAPK (26). The phosphorylation of P38 increased the apoptosis of PANC-1 and SW1990 PC cells. P38 is mainly activated by a variety of stimuli, such as oxidative stress, UV irradiation and osmotic pressure changes, mainly to induce apoptosis (28). In addition to MAPKs, another protein kinase, AKT, activates a range of downstream substrates through phosphorylation, including the B-cell lymphoma 2-associated death promoter, glycogen synthase kinase $3 \beta$ and forkhead box $\mathrm{O}$ transcription factors, which mediate cell proliferation (29). The present study observed that EVO downregulated p-ERK in a dose-dependent manner. ERK serves an important role in cell proliferation and differentiation and is generally considered to be a regulator of cell survival (30). The results of the present study indicated that AKT and ERK were constitutively activated in PC cells and that the levels of p-AKT and p-ERK were significantly downregulated following EVO treatment in a dose-dependent manner. Previously reported data show that PC cells are protected by autophagy under extreme conditions (31). In the present study, it was hypothesized that EVO mediates autophagy in human PC cells. STAT3 is constitutively activated and its prognostic value has been associated with p-STAT3 
signatures in PC (32). Therefore, targeting STAT3 signaling with small molecule inhibitors is an emerging therapeutic strategy for PC (33). Mechanistic studies have indicated that EVO inhibits the formation of p-STAT3, leading to decreased autophagy. Under normal physiological conditions, STAT3 has low expression levels (34). Abnormal levels of p-STAT3 transform normal fibrotic cells, showing the role of protooncogenes, which are involved in tumor cell proliferation, differentiation, invasion, metastasis and angiogenesis (35). Published studies show that silencing STAT3 genetically or pharmacologically reduced the expression of LC3 in Capan-2 cells, suggesting that STAT3 transcriptionally regulates LC3 (36). Furthermore, silencing STAT3 increased p62 levels, suggesting that STAT3 positively regulates the activation of autophagy (36). In addition, the present study found that EVO inhibited the phosphorylation of STAT3 in PC cells, whereas expression of the autophagy-related protein LC3 was downregulated and that of p62 was upregulated. It was found that EVO promoted the apoptosis of PC cells, whereas autophagy was suppressed. In a nutrient-poor environment, PC cell lines have been reported to exhibit autophagy, which supports cell survival (37). The analysis of human surgical specimens suggested higher autophagy activity in cancer tissues than in non-cancerous tissues (38). PC cell resistance has often been associated with self-autophagy (31). Therefore, autophagy is a mechanism that not only enables cells to adapt to hypoxic environments but also supports the survival of the entire microenvironment of the PC tissue. Therefore, autophagy is a favorable therapeutic target (39). Cells prevent apoptosis through mitochondrial autophagy, which is a selective autophagic mechanism that maintains the intracellular redox state (40). Under hypoxic conditions, PC cells can evade death through autophagy (31). In the present study, autophagy formation was induced in PC cells through a nutrient-deficient medium (17). The results revealed that EVO inhibited autophagy in PC cells and thus reduced the self-protection of PC cells. An orthotopic pancreas tumor model was established in nude mice, and the growth of pancreatic tumors was close to that in the human physiological environment. 18F-FDG-PET has high sensitivity and specificity in the detection of PC in patients with a suspected pancreatic mass. After 4 weeks of EVO treatment of the nude mice, micro-PET imaging revealed that the tumor mass was significantly lower than that of the untreated control group. Regional uptake of the tracer was assessed using the ROI method and SUVs. We found that as the concentration of EVO increased, the body weight of nude mice increased correspondingly compared with the control group, which also indicated that EVO also serves a role in the health effects of nude mice while inhibiting tumor growth. It was found that, with increasing drug concentration, the size and volume of the tumors were increasingly smaller, which was consistent with the in vitro experimental results. In addition, the immunohistochemistry revealed that the expression of P-AKT in the tumor tissues was decreased concentration-dependently, which was also consistent with the in vitro experiments. EVO inhibited cell migration similarly in the two cell lines, which is similar to a previous study by Ogasawara $e t$ al showing that EVO inhibited lung cancer invasion and cell migration (41).

In conclusion, the present study demonstrated that EVO exhibited potent antitumor effects on human PC cells and promoted their apoptosis by inhibiting the phosphorylation of PI3K/AKT and MEK/ERK pathway proteins. Furthermore, EVO promoted PC cell growth retardation by inhibiting the phosphorylation of STAT3, reducing phagocytosis. In the absence of nutrient deficiency, PC cells survive by autophagy. For the first time, to the best of our knowledge, EVO was found to inhibit the formation of autophagy in PC cells, thereby destroying the self-protection mechanism of PC cells. Based on these results, EVO has potential therapeutic effects that warrant further investigation for development and possible clinical trials for PC.

\section{Acknowledgements}

Not applicable.

\section{Funding}

This study was supported by funding from Zhejiang Provincial Administration of Traditional Chinese Medicine Youth Fund Project (grant no. 2014ZQ020) and the Natural Science Foundation of Zhejiang Project (grant nos. LQ18H290003 and Y190029H46). Funding was also received from Wenzhou Science and Technology Project (grant no. Y20160052).

\section{Availability of data and materials}

The datasets used and/or analyzed during the current study are available from the corresponding author on reasonable request.

\section{Authors' contributions}

$\mathrm{ZH}$ and PZ contributed to the conception of the study; HC, ZW and YL contributed significantly to analysis and manuscript preparation; BZ, JW and MBJ performed the data analyses and wrote the manuscript; QZ and HT helped perform the analysis with constructive discussions.

\section{Ethics approval and consent to participate}

This study was approved by the Laboratory Animal Ethics Committee of Wenzhou Medical University and Laboratory Animal Centre of Wenzhou Medical University (Wenzhou, China).

\section{Patient consent for publication}

Not applicable.

\section{Competing interests}

The authors declare that they have no competing interests.

\section{References}

1. Chen W, Zheng R, Baade PD, Zhang S, Zeng H, Bray F, Jemal A, Yu XQ and He J: Cancer statistics in China, 2015. CA Cancer J Clin 66: 115-132, 2016.

2. Stathis A and Moore MJ: Advanced pancreatic carcinoma: Current treatment and future challenges. Nat Rev Clin Oncol 7: 163-172, 2010.

3. Teague A, Lim KH and Wang-Gillam A: Advanced pancreatic adenocarcinoma: A review of current treatment strategies and developing therapies. Ther Adv Med Oncol 7: 68-84, 2015. 
4. Zhan HX, Xu JW, Wu D, Wu ZY, Wang L, Hu SY and Zhang GY: Neoadjuvant therapy in pancreatic cancer: A systematic review and meta-analysis of prospective studies. Cancer Med 6: 1201-1219, 2017

5. Long J, Zhang Y, Yu X, Yang J, LeBrun DG, Chen C, Yao Q and Li M: Overcoming drug resistance in pancreatic cancer. Expert Opin Ther Targets 15: 817-828, 2011.

6. Yusufi M, Banerjee S, Mohammad M, Khatal S, Venkateswara Swamy K, Khan EM, Aboukameel A, Sarkar FH and Padhye S: Synthesis, characterization and anti-tumor activity of novel thymoquinone analogs against pancreatic cancer. Bioorg Med Chem Lett 23: 3101-3104, 2013.

7. Su J, Zhou X, Wang L, Yin X and Wang Z: Curcumin inhibits cell growth and invasion and induces apoptosis through down-regulation of Skp2 in pancreatic cancer cells. Am J Cancer Res 6: 1949-1962, 2016

8. Liu AJ, Wang SH, Chen KC, Kuei HP, Shih YL, Hou SY, Chiu WT, Hsiao SH and Shih CM: Evodiamine, a plant alkaloid, induces calcium/JNK-mediated autophagy and calcium/mitochondria-mediated apoptosis in human glioblastoma cells. Chem Biol Interact 205: 20-28, 2013.

9. Zhou X, Ren F, Wei H, Liu L, Shen T, Xu S, Wei J, Ren J and Ni H: Combination of berberine and evodiamine inhibits intestinal cholesterol absorption in high fat diet induced hyperlipidemic rats. Lipids Health Dis 16: 239, 2017.

10. Schwarz NA, Spillane M,La Bounty P, Grandjean PW,Leutholtz B and Willoughby DS: Capsaicin and evodiamine ingestion does not augment energy expenditure and fat oxidation at rest or after moderately-intense exercise. Nutr Res 33: 1034-1042, 2013.

11. Zhang YN, Yang YF and Yang XW: Blood-brain barrier permeability and neuroprotective effects of three main alkaloids from the fruits of Euodia rutaecarpa with MDCK-pHaMDR cell monolayer and PC12 cell line. Biomed Pharmacother 98: 82-87, 2018.

12. Kim SH, Kang JG, Kim CS, Ihm SH, Choi MG and Lee SJ: Evodiamine Suppresses Survival, Proliferation, Migration and Epithelial-Mesenchymal Transition of Thyroid Carcinoma Cells. Anticancer Res 38: 6339-6352, 2018.

13. Guo XX, Li XP, Zhou P, Li DY, Lyu XT, Chen Y, Lyu YW, Tian K, Yuan DZ, Ran JH, et al: Evodiamine Induces Apoptosis in SMMC-7721 and HepG2 Cells by Suppressing NOD1 Signal Pathway. Int J Mol Sci 19: 19, 2018.

14. Tu YJ, Fan X, Yang X, Zhang C and Liang HP: Evodiamine activates autophagy as a cytoprotective response in murine Lewis lung carcinoma cells. Oncol Rep 29: 481-490, 2013.

15. Han S, Woo JK, Jung Y, Jeong D, Kang M, Yoo YJ, Lee $H$, Oh SH, Ryu JH and Kim WY: Evodiamine selectively targets cancer stem-like cells through the p53-p21-Rb pathway. Biochem Biophys Res Commun 469: 1153-1158, 2016.

16. Jiang J and Hu C: Evodiamine: A novel anti-cancer alkaloid from Evodia rutaecarpa. Molecules 14: 1852-1859, 2009.

17. Kim SE, Park HJ, Jeong HK, Kim MJ, Kim M, Bae ON and Baek SH: Autophagy sustains the survival of human pancreatic cancer PANC-1 cells under extreme nutrient deprivation conditions. Biochem Biophys Res Commun 463: 205-210, 2015.

18. Pratesi G, Petrangolini G, Tortoreto M, Addis A, Belluco S, Rossini A,SelleriS,RumioC,Menard S and Balsari A:Therapeutic synergism of gemcitabine and $\mathrm{CpG}$-oligodeoxynucleotides in an orthotopic human pancreatic carcinoma xenograft. Cancer Res 65: 6388-6393, 2005

19. Wei WT, Chen H, Wang ZH, Ni ZL, Liu HB, Tong HF, Guo HC, Liu DL and Lin SZ: Enhanced antitumor efficacy of gemcitabine by evodiamine on pancreatic cancer via regulating PI3K/Akt pathway. Int J Biol Sci 8: 1-14, 2012.

20. Jemal A, Siegel R, Ward E, Hao Y, Xu J and Thun MJ: Cancer statistics, 2009. CA Cancer J Clin 59: 225-249, 2009.

21. Burris HA III, Moore MJ, Andersen J, Green MR, Rothenberg ML, Modiano MR, Cripps MC, Portenoy RK Storniolo AM, Tarassoff P, et al: Improvements in survival and clinical benefit with gemcitabine as first-line therapy for patients with advanced pancreas cancer: A randomized trial. J Clin Oncol 15: 2403-2413, 1997.

22. Wang C, Li S and Wang MW: Evodiamine-induced human melanoma A375-S2 cell death was mediated by PI3K/Akt/caspase and Fas-L/NF-kappaB signaling pathways and augmented by ubiquitin-proteasome inhibition. Toxicol In Vitro 24: 898-904, 2010 .
23. Yotsumoto F, Fukami T, Yagi H, Funakoshi A, Yoshizato T, Kuroki $\mathrm{M}$ and Miyamoto S: Amphiregulin regulates the activation of ERK and Akt through epidermal growth factor receptor and HER3 signals involved in the progression of pancreatic cancer. Cancer Sci 101: 2351-2360, 2010.

24. Rasul A, Yu B, Zhong L, Khan M, Yang H and Ma T: Cytotoxic effect of evodiamine in SGC-7901 human gastric adenocarcinoma cells via simultaneous induction of apoptosis and autophagy. Oncol Rep 27: 1481-1487, 2012.

25. Liu AJ, Wang SH, Hou SY, Lin CJ, Chiu WT, Hsiao SH, Chen TH and Shih CM: Evodiamine Induces Transient Receptor Potential Vanilloid-1-Mediated Protective Autophagy in U87-MG Astrocytes. Evid Based Complement Alternat Med 2013: 354840, 2013.

26. Wang R, Deng D, Shao N, Xu Y, Xue L, Peng Y, Liu Y and Zhi F: Evodiamine activates cellular apoptosis through suppressing PI3K/AKT and activating MAPK in glioma. Onco Targets Ther 11: 1183-1192, 2018.

27. Carnero A and Paramio JM: The PTEN/PI3K/AKT Pathway in vivo, Cancer Mouse Models. Front Oncol 4: 252, 2014.

28. Zhuang S and Schnellmann RG: A death-promoting role for extracellular signal-regulated kinase. J Pharmacol Exp Ther 319: 991-997, 2006

29. Yin S, Yang S, Pan X, Ma A, Ma J, Pei H, Dong Y, Li S, Li W and Bi X: MicroRNA 155 promotes ox LDL induced autophagy in human umbilical vein endothelial cells by targeting the PI3K/Akt/mTOR pathway. Mol Med Rep 18: 2798-2806, 2018.

30. Dhillon AS, Hagan S, Rath O and Kolch W: MAP kinase signalling pathways in cancer. Oncogene 26: 3279-3290, 2007.

31. Bellot G, Garcia-Medina R, Gounon P, Chiche J, Roux D, Pouysségur $\mathrm{J}$ and Mazure NM: Hypoxia-induced autophagy is mediated through hypoxia-inducible factor induction of BNIP3 and BNIP3L via their BH3 domains. Mol Cell Biol 29: 2570-2581, 2009.

32. Mace TA, Ameen Z, Collins A, Wojcik S, Mair M, Young GS, Fuchs JR, Eubank TD, Frankel WL, Bekaii-Saab T, et al: Pancreatic cancer-associated stellate cells promote differentiation of myeloid-derived suppressor cells in a STAT3-dependent manner. Cancer Res 73: 3007-3018, 2013.

33. Hu Y, Zhao C, Zheng H, Lu K, Shi D, Liu Z, Dai X, Zhang Y, Zhang X, Hu W, et al: A novel STAT3 inhibitor HO-3867 induces cell apoptosis by reactive oxygen species-dependent endoplasmic reticulum stress in human pancreatic cancer cells. Anticancer Drugs 28: 392-400, 2017.

34. Szczepanek K, Lesnefsky EJ and Larner AC: Multi-tasking: Nuclear transcription factors with novel roles in the mitochondria. Trends Cell Biol 22: 429-437, 2012.

35. Aigner P, Just V and Stoiber D: STAT3 isoforms: Alternative fates in cancer? Cytokine 118: 27-34, 2019.

36. Gong J, Muñoz AR, Chan D, Ghosh R and Kumar AP: STAT3 down regulates LC3 to inhibit autophagy and pancreatic cancer cell growth. Oncotarget 5: 2529-2541, 2014

37. Azad MB, Chen Y, Henson ES, Cizeau J, McMillan-Ward E, Israels SJ and Gibson SB: Hypoxia induces autophagic cell death in apoptosis-competent cells through a mechanism involving BNIP3. Autophagy 4: 195-204, 2008.

38. Sullivan R, Paré GC, Frederiksen LJ, Semenza GL and Graham $\mathrm{CH}$ : Hypoxia-induced resistance to anticancer drugs is associated with decreased senescence and requires hypoxia-inducible factor-1 activity. Mol Cancer Ther 7: 1961-1973, 2008

39. Greene LM, Nolan DP, Regan-Komito D, Campiani G, Williams DC and Zisterer DM: Inhibition of late-stage autophagy synergistically enhances pyrrolo-1,5-benzoxazepine-6-induced apoptotic cell death in human colon cancer cells. Int J Oncol 43: 927-935, 2013.

40. Blommaart EF, Luiken JJ, Blommaart PJ, van Woerkom GM and Meijer AJ: Phosphorylation of ribosomal protein S6 is inhibitory for autophagy in isolated rat hepatocytes. J Biol Chem 270: 2320-2326, 1995.

41. Ogasawara M, Matsubara T and Suzuki H: Inhibitory effects of evodiamine on in vitro invasion and experimental lung metastasis of murine colon cancer cells. Biol Pharm Bull 24: 917-920, 2001.

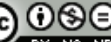

This work is licensed under a Creative Commons Attribution-NonCommercial-NoDerivatives 4.0 International (CC BY-NC-ND 4.0) License. 